\title{
EL APRENDIZAJE AUTORREGULADO
}

\section{SELF-REGULATED LEARNING}

\section{LUISA NOA SILVERIO}

Facultad de Educación a Distancia. Unviversidad de La Habana, Cuba. luisanoa@fed.uh.cu

RESUMEN

Las nuevas oportunidades de acceder a los recursos que proporciona Internet, permite que las personas puedan adquirir conocimientos de manera informal en campos disímiles del saber. Por otro lado, el crecimiento de forma exponencial del conocimiento requiere de todo ciudadano estar preparado para un aprendizaje continuo a lo largo de la vida. Las universidades deben crear las condiciones para que en sus programas se considere la formación de competencias que permitan a los egresados emprender de forma autónoma su futura superación. El presente trabajo analiza las investigaciones llevadas a efecto en la Facultad de Educación a Distancia de la Universidad de La Habana en el uso de herramientas sociales donde solamente se ha analizado si ha sido posible la apropiación de nuevos conocimientos, pero no se ha investigado la forma o los métodos empleados por los estudiantes de los programas de postgrado. Se propone los pasos de un proyecto de investigación para poder discernir cómo sería posible diseñar el aprendizaje para que incluya las nuevas competencias de autoregulación del aprendizaje.

PALABRAS CLAVE: aprendizaje autoregulado, nuevos espacios de aprendizaje, aprendizaje, metacognición, educación a distancia, teoría de aprendizaje, competencias.
New opportunities to access resources that provide Internet allows people to acquire skills informally in disparate fields of knowledge. On the other hand, exponential growth of knowledge requires every citizen to be prepared for continuous learning throughout life. Universities must create the conditions for its programs in skills training that should allow the graduates to undertake autonomously their future improvement. This paper analyzes the research at the Faculty of Distance Education of the University of Havana in the use of social tools where only analyzed whether it was possible the appropriation of new knowledge is discussed, but has not been investigated how or methods used by students in posgraduate programs. The steps of a research project is proposed to discern how it would be possible to design the learning including the new competencies of self-learning.

KEYWORDS: self-regulated learning, new learning spaces, learning, metacognition, distance learning, learning theory, skills. 
INTRODUCCIÓN

Existen muchas diferencias entre un ambiente electrónico o virtual de aprendizaje y un espacio real, tradicional donde transcurren los sucesos y los eventos educativos --como son las aulas, los anfiteatros, laboratorios, etc.--. En los ambientes virtuales la ausencia de información no verbal -gestos, tono de voz, lenguaje corporal, etc. --, así como la diferencia en la percepción del tiempo, incluyen nuevos factores que determina la selección de una estrategia de instrucción, es decir, de una estrategia de enseñanza-aprendizaje. Por otra parte, la falta de intelección, de experiencias de cómo se aprende en estos nuevos espacios y de una cultura en el uso de estos medios, hacen que tanto profesores como estudiantes, reflejen cierta "inercia" para producir los cambios necesarios.

La complejidad de los ambientes de aprendizaje, la exigencia de la personalización en la enseñanza, la autonomía -cada vez mayor-del sujeto que aprende, la posibilidad del acceso a las fuentes de información y a los recursos educativos, así como los cambios tecnológicos en que se sustentan el flujo de información y las comunicaciones, hacen cada vez más necesarias la investigación, las propuestas de cambio en el diseño y la configuración de los sistemas de acciones para el aprendizaje.

Dron y Anderson (2014) citan a otros autores sobre la idea de que las redes son el lenguaje de nuestros tiempos pero nuestras instituciones no están programadas para entenderlo. Este aspecto es de tal importancia que es necesario recordar la vinculación entre el medio y el mensaje. Los medios utilizados por los educadores tienen un profundo efecto sobre el contenido que se enseña, la organización del proceso de aprendizaje y el rango de las actividades de aprendizaje que sellevan a efecto. Los nuevos medios han alterado, de forma radical las condiciones para la enseñanzaaprendizaje.

Es de señalar que la etapa actual se caracteriza por la conectividad y por el amplio rango de las relaciones que se concretan mediante los nuevos medios. Las cifras de las personas que frecuentan los espacios sociales virtuales pueden catalogarse de astronómicas. Todo ello implica nuevas formas de pensar, nuevas formas de representar el mundo en que vivimos.

Por otro lado, Zimmerman (2008) señala que el tema de cómo los estudiantes se convierten en sujetos que auto regulan su aprendizaje, ha sido un tema de investigación importante durante décadas. Este autor señala que estamos ya en una segunda ola de investigaciones en este campo que ha incluido el desarrollo de mediciones en línea del proceso de auto regulación, los aspectos motivacionales y el reconocimiento del aprendizaje en contextos auténticos. Los nuevos métodos de investigación incluyen trazas en las computadoras, diarios de estudio y microanálisis.

\section{LAS TEORÍAS DE APRENDIZAJE SOCIAL}

Las nuevas propuestas de comprensión de cómo el hombre aprende, están impactadas por el uso de estas tecnologías y en su centro están las formas sociales de aprendizaje.

Dron y Anderson (2014) plantean que hasta hace poco la mayoría de la literatura en aprendizaje social asumía que la interacción entre participantes tomaba lugar cara-a-cara, o sea de forma presencial. Hoy, en la literatura pedagógica, especialmente en educación a distancia y en la perspectiva del e-learning se asume un contexto mediado electrónicamente para la enseñanza-aprendizaje. Entre las teorías sociales del aprendizaje, estos autores señalan: el constructivismo social, la era conectivista que incluye la heutagogía, la cognición distribuida, la teoría de actividad, la teoría de la complejidad y la teoría del conectivismo, donde se incluyen las comunidades de práctica y el conectivismo de Siemens. Estas son teorías que tratan de dar una explicación a las formas de aprender de nuestro tiempo, donde se parte de la necesidad del aprendizaje a lo largo de la vida, que el individuo adquiera la capacidad de aprender a aprender y donde se mezclan el aprendizaje formal e informal.

Las tecnologías que nos proveen de espacios virtuales para la comunicación y sitios de encuentros sociales, desde el correo electrónico hasta los sistemas gestores de aprendizaje (plataformas), son ubicuas en nuestras escuelas y universidades. Por lo que se produce, en gran escala, las facilidades para el aprendizaje intencional o no intencional.

No es de extrañar que las reflexiones y las producciones teóricas traten de captar las formas, las condiciones y peculiaridades en que se produce el aprendizaje en nuestros días. Si aspiramos que las investigaciones educativas tengan la validez necesaria, será necesario conocer de cerca estas producciones teóricas. 
LAS NUEVAS HERRAMIENTAS DE COMUNICACIÓN Y EL PROCESO DE APRENDIZAJE

En la facultad de Educación a Distancia de la Universidad de La Habana hemos desarrollado investigaciones sobre las herramientas sociales. Desde que se comenzó a utilizar las plataformas o gestores para cursos en línea, en el 1999, la Facultad ha realizado varias investigaciones sobre cómo los estudiantes se comportan en dicho medio. Al principio se utilizaron las herramientas sociales de comunicación denominadas de primera generación: email, chat y foros de discusión. Los estudiantes siempre han considerado el foro de discusión como una buena herramienta de intercambio entre los pares, entre ellos y sus profesores. En la investigación realizada con foros de discusión en varios grupos de estudiantes de los programas de postgrado (Noa, 2014) se analizaron los mensajes enviados (análisis de contenido) y se clasificaron según una tabla de indicadores para conocer si en el desarrollo del foro, cuando se llegaba a su término, se había construido conocimiento.

Para este estudio se utilizó el software ATLAS.ti. Este paquete está destinado al procesamiento y análisis de datos cualitativos y puede considerarse como de segunda generación de CAQDAS, siglas que inglés significa: "computer assisted qualitative data analysis“

Se llegó a la conclusión que en los foros estudiados se llegaba solamente a una etapa de intercambio de ideas y de opiniones, que los estudiantes hacían referencia a algunos elementos extraídos de las teorías estudiadas. El objetivo central de esta investigación era determinar los resultados del proceso de cognición cuando se utilizaban los foros.

El resultado final de este estudio indicó que mediante las discusiones se había realizado un intercambio de ideas sobre los temas analizados ,pero que no se había producido construcción de tesis o hipótesis. No obstante, la evaluación de los estudiantes y su grado de satisfacción fue muy positiva porque para ellos la posibilidad de comunicarse había sido una experiencia enriquecedora. Nunca nos planteamos en qué medida esas razones reflejaban algún otro beneficio que nosotros no habíamos considerado, por ejemplo, la motivación al estudio.

De igual modo resultó la investigación en la construcción de los Wiki (Noa, 2014) como segunda generación de herramientas sociales. Los Wiki han revolucionado la forma de concebir esos espacios de creación colectiva y constituyen una herramienta capaz de soportar la colaboración en un gran número de actividades y de creación de contenidos, así como constituyen una forma para la realización de experiencias de aprendizaje.

Utilizar los Wiki requiere de los participantes ciertas habilidades para orientarse y utilizar las opciones que permiten la colaboración entre ellos. El Wiki hoy en día es uno de los nuevos espacios que configuran la Web $2.0 \mathrm{y}$ por la forma abierta de crear contenidos, se ha convertido en una herramienta ideal para las diferentes manifestaciones del movimiento de conocimiento abierto. Así tenemos diferentes proyectos que utilizan el Wiki como son los Wikibooks, Wikitutorials, Wikipedia, Wikicourse, Lyricwiki y Wikiversity.

En la investigación se habilitaron 4 grupos de estudiantes. No todos los integrantes de los grupos constituidos participaron. Los grupos más exitosos han sido aquellos que han contado con responsables activos, que han sabido estimular la participación.

En la primera investigación de trabajo con los Wiki, se adquirió experiencias importantes de cómo establecer pautas de elaboración de los Wiki y las formas de conducirlos. Se valoró la calidad de los textos construidos, los grados de satisfacción de los estudiantes, las opiniones de los responsables de los grupos participantes y las conductas no deseables por parte de los estudiantes. Y se consideró que la experiencia fue muy enriquecedora. Para los estudiantes, tanto en el primer experimento como en el segundo, se encontró que se realizaron búsquedas, selección de textos y mezclas de los mismos, bajo una nueva lógica creada para exponer los contenidos. Pero tampoco se consideró en esta investigación si existió otro tipo de aprendizaje que no fuera la de los objetivos propuestos en el aprendizaje de contenidos. Es decir, si la experiencia desarrollada les permitió adquirir conocimientos sobre como planificar la actividad y cómo hacer las búsquedas, seleccionar los artículos, tomar notas y las formas de cómo se ha organizado en la agenda personal el trabajo realizado.

\section{EL APRENDIZAJE AUTORREGULADO}

Entre los principios que sustentan los programas de postgrado de la Facultad de Educación a Distancia están: la independencia y autonomía de los que aprenden (sujeto activo), la naturaleza social del proceso de aprendizaje, la flexibilidad y la interactividad. Dos de ellos son de vital importancia en el aprendizaje del adul- 
to: la independencia y autonomía, así como la naturaleza social del aprendizaje.

Este enfoque sugiere que el sujeto está condicionado por factores externos como los sociales, políticos y económicos, y factores internos como son los biológicos, emocionales y cognitivos. Desde esta perspectiva el aprendizaje autorregulado es considerado como una interacción entre los sujetos y los elementos ambientales (Chaves-Barboza y Trujillo-Torres, 2015). El sujeto a la vez que adquiere conocimientos, desarrolla determinadas habilidades para mantener y mejorar el propio proceso de aprendizaje. Adquiere determinadas habilidades metacognitivas para establecer, evaluar y modificar sus propias metas, su comportamiento y las estrategias de aprendizaje.

El aprendizaje auto-regulado es un proceso cíclico dividido en tres etapas: la previsión, ejecución, y auto-reflexión (Chaves-Barboza y Trujillo-Torres, 2015). En la fase de previsión, el sujeto establece sus objetivos de aprendizaje y las estrategias correspondientes a tales metas. En la fase de ejecución, el sujeto pone en práctica las estrategias planeadas en la etapa anterior y realizan actividades de autocontrol. Y por último, en la etapa de auto-reflexión, el sujeto utiliza toda la información obtenida y las experiencias de la etapa anterior con el fin de ajustar sus estrategias y reiniciar el ciclo de aprendizaje autorregulado. El éxito alcanzado lleva a una especie de estimulación y propicia la motivación para emprender el próximo ciclo de aprendizaje autorregulado.

En la literatura se reconoce la importancia de las investigaciones en el aprendizaje autorregulado, así como la necesidad de estimular en los estudiantes la adquisición de las competencias que permitan desplegar las iniciativas para un aprendizaje autónomo. No es necesario recordar que nuestra época nos impone un aprendizaje a lo largo de la vida, así como enfrentar problemas complejos en cuya solución se requiere utilizar las competencias adquiridas para el aprendizaje autorregulado.

LOS ENTORNOS PERSONALES DE APRENDIZAJE (PLE) En el marco teórico del aprendizaje autorregulado, se presta especial atención a las circunstancias personales del sujeto y los factores ambientales y tecnológicos (Chaves-Barboza y Trujillo-Torres, 2015). "Sea cual sea la época en la que nos situemos, las personas han tenido siempre un entramado de conexiones sociales y de fuentes básicas de las que aprender. Ese "entramado" ha estado condicionado siempre por las fuentes de conocimiento fiable de las que disponían y de las cuales se entendía que debían aprender. Así, en un primer momento el entorno de aprendizaje se limitaba a la tribu y a la familia, posteriormente incluyó también a un maestro del que éramos aprendices, con la aparición y proliferación de los libros incluyó a los libros y, cuando aparece la escuela, centraliza en ella casi todos sus elementos.

Siempre hemos tenido un entorno personal del que aprendemos, aunque es probable que no hayamos sido conscientes de él... "(Castañeda, L. y Adell, 2013).

Loa entornos personales de aprendizaje y las ideas educativas relacionadas con estos entornos son útiles para estudiar cómo la forma de aprender puede verse afectado por las tecnologías que son fáciles de usar y ubicuas. Estas tecnologías que son cada vez más extendidas a todas las actividades humanas, y a todas las regiones del planeta. Ellas están integradas a la vida cotidiana y permiten su adaptación a necesidades, gustos y preferencias de los individuos.

La gestión de todas las herramientas tecnológicas para el aprendizaje gestionado por el propio aprendiz y el espacio generado para ello, es lo que se reconoce como PLE (Personal Learning Environment-- en inglés). El aprendizaje dentro de este entorno requiere un sujeto activo y reflexivo. Por otra parte, reconocer la existencia de estos espacios implica para el aprendiz, la realización de tareas autorreguladas y de aquellas dentro de lo que se reconoce como metacognición. Esto quiere decir el establecimiento de objetivos, la elección y el manejo de sus propios contenidos, el establecimiento de comunicación con otros estudiantes y maestros durante sus gestiones y, finalmente, el logro de sus metas de aprendizaje (Chaves-Barboza y Trujillo-Torres, 2015).

\section{LOS ESPACIOS SOCIALES DE APRENDIZAJE (MEDIOS SOCIALES)}

Dabbagh y Kitsantas (Dabbagh y Kitsantas, 2011) indican que los "medios de comunicación social" es un término del Siglo XXI y que se utiliza para definir una variedad de herramientas en red o tecnologías que hacen hincapié en los aspectos sociales de Internet como canal para la comunicación, la colaboración y la expresión creativa. Estos autores también subrayan que las instituciones de educación superior deberían integrar las plataformas de medios sociales que permiten la creación de espacios de aprendizaje 
personales y sociales. Para ello elaboraron un marco pedagógico basados en las tres etapas del modelo para el aprendizaje autorregulado de Zimmerman: la previsión, ejecución, y autoreflexión y de esta forma tratan de combinar el trabajo en espacios personales y sociales de aprendizaje.

Estemarco pedagógico (Dabbagh y Kitsantas, 2011) consta de tres niveles: (1) Gestión de información personal, (2) la interacción social y la colaboración, y (3) la agregación y la gestión de la información. Este último nivel se trata que los estudiantes reflexionen sobre su proceso de aprendizaje.

\section{PROYECTO DE INVESTIGACIÓN}

Para todo educador es necesario comprender como las personas gestionan su aprendizaje, y a su vez, es importante también delimitar cómo es posible adquirir las competencias que aseguren una eficiente autorregulación del aprendizaje. Los dos aspectos indicados con anterioridad: los factores internos vinculados al sujeto y los factores externos vinculados al medio social: lo político y económico y cultural. Estos se entremezclan y presentan un complejo entramado de conexiones tanto en los espacios personales de aprendizaje como en los sociales.

El marco pedagógico que se asume es el de Dabbagh y Kitsantas (2011) donde se reconocen tres etapas.

Un objetivo central es dilucidar la influencia que tienen los colectivos de estudio y trabajo para estimular las actividades autorregulatorias del aprendizaje. En una primera etapa se deben seleccionar los grupos de estudiantes y propiciar trabajos colaborativos mediante los espacios virtuales de intercambio y se deben estimular la creación y utilización de espacios personales.

Los profesores tendrían el papel de asesores o guías para el proceso de aprendizaje y un aspecto esencial de su trabajo es aprovechar todas las oportunidades para orientar las estrategias de autocontrol y gestión del aprendizaje en los estudiantes, es decir propiciar de esta forma la creación de las competencias necesarias para la autorregulación del aprendizaje. Estas competencias deben ser claramente definidas y vinculadas a las variables de investigación.

Aplicar técnicas de observación, analizar las trazas dejadas en el software de comunicación, análisis de los contenidos de los mensajes, entrevistas individuales y grupales y cuadernos de trabajo. También, se debe propiciar que los participantes elaboren notas de sus actividades y si es posible, sus reflexiones. Las variables que se asumirían: auto-evaluación, organización de sus actividades, organización del tiempo, objetivos o metas trazadas, planificación, metodología empleada para la búsqueda de información, selección de información y su organización; estructuración de su ambiente de trabajo o entorno personal de aprendizaje, búsqueda de asistencia o ayuda, el trabajo en equipo y su organización personal, interés y conocimiento sobre cómo otras personas organizan su trabajo; y por último, aplicar test para medir el grado de satisfacción de los estudiantes.

CONCLUSIONES

En las condiciones actuales se requiere que las universidades gradúen profesionales que gestionen su propio conocimiento.

Los estudios que se realicen sobre este tema deben estar centrados en las vías que los estudiantes construyen sus estrategias de autorregulación del aprendizaje y cómo adquieren las competencias necesarias para ello.

Se desprende la importancia de la realización de investigaciones que nos ubiquen en la formación de nuestros estudiantes relacionada con la autogestión de su aprendizaje, así como las posibilidades de los profesores de asumir estos roles. Estas investigaciones darán pautas para la retroalimentación del sistema educativo y la posible toma de decisiones.

Las instituciones educativas deben propiciar el intercambio en estos estudios, así como brindar oportunidad para la superación de sus profesores mediante diversos recursos como son eventos y espacios sociales de comunicación para el intercambio de experiencias. 


\section{REFERENCIAS BIBLIOGRÁFICAS}

Castañeda, L. y Adell, J. (Eds.) Entornos Personales de Aprendizaje: claves para el ecosistema educativo en red. Alcoy: Marfil. 2013.

Chaves-Barboza,E., Trujillo-Torres, J. y LópezNúñez, J.A. Accomplishments in Learning. Self-Regulation in Personal Environments. Creative Education, 2015, 6, pp. 1108-1120.

Dabbagh, N., \& Kitsantas, A., Personal Learning Environments, social media, and selfregulated learning: A natural formula for connecting formal and informal learning, Internet and Higher Education (2011), doi:10.1016/j.iheduc.2011.06.002

Dron, J. and Anderson, Teaching Crowds, Learning and Social Media. Athabasca University Press. 2014. ISBN: 978-1-927356-81-4. En: http:// aupress.ca/index.php/books/120235

Noa, L. Los Foros Electrónicos de discusión. Un estudio a través del análisis de contenido. Evento Internacional Inforedu, Informática 2004. La Habana.

Noa, L. Wiki para el aprendizaje. En el libro: Las TIC y la Calidad en la Educación Superior, 2014. Editora de la Universidad ECOTEC, Guayaquil, Ecuador.

Zimmerman, B.J. Investigating Self-Regulation and Motivation: Historical Background, Methodological Developments, and Future Prospects. American Educational Research Journal Math 2008, Vol. 45, No. 1, pp. 166 -183. DOI: 10.3102/0002831207312909 